\title{
QUALIDADE ESTRUTURAL DO SOLO APÓS O CORTE FLORESTAL POR HARVESTER DE PNEUS E ESCAVADEIRA HIDRÁULICA ADAPTADA
}

\author{
SOIL STRUCTURAL QUALITY AFTER FOREST CUTTING BY RUBBER-TIRES AND \\ EXCAVATOR-BASED HARVESTERS \\ Leandro Tonhato ${ }^{1}$, Eduardo da Silva Lopes ${ }^{2}$, Carla Krulikowski Rodrigues ${ }^{3}$, Jean Alberto \\ Sampietro ${ }^{4}$, Allan Libanio Pelissari ${ }^{5}$, Matheus Kaminski Cândido da Silva ${ }^{6}$ \\ 1, 2,6 Universidade Estadual do Centro-Oeste, Irati, Paraná, Brasil-leandro_tonhato@hotmail.com, \\ eslopes@unicentro.br\&matheuskcs@gmail.com \\ 3, 5 Universidade Federal do Paraná, Curitiba, Paraná, Brasil - carlakr@gmail.com \& \\ allanpelissari@ufpr.br \\ ${ }^{4}$ Universidade Estadual de Santa Catarina, Lages, Santa Catarina, Brasil-jean.sampietro@udesc.br
}

\section{RESUMO}

O objetivo foi avaliar a qualidade estrutural do solo após corte florestal com harvester de pneus e escavadeira hidráulica adaptada em LATOSSOLO VERMELHO distrófico típico. O experimento foi delineado de forma inteiramente casualizada com instalação de quatro unidades amostrais com três pontos de repetição. Além disso, foram coletadas amostras de solo na trilha de tráfego dos rodados da máquina nas camadas de 0 a 0,$10 ; 0,11$ a 0,$20 ; 0,21$ a 0,30 ; e 0,31 a 0,40 m de profundidade para determinação dos parâmetros de densidade e porosidade total e, também, foi mensurada a resistência do solo à penetração na trilha dos rodados e entre rodados. Dessa forma, foi possível determinar que a densidade e a porosidade total média antes do tráfego das máquinas foram de $1,10 \mathrm{~g} \mathrm{~cm}^{-3}$ e $53,85 \%$, respectivamente, ocorrendo efeitos significativos nessas variáveis após o tráfego do harvester de pneus em todas as camadas avaliadas. Quanto a resistência do solo à penetração, verificou-se que houve aumento significativo na trilha dos rodados do harvester, tanto de pneus como de escavadeira, contudo, havendo incremento de cerca de $77 \%$ na camada de 0,10 m após tráfego do harvester de pneus.

PALAVRAS-CHAVE: Colheita de madeira, Física do Solo, Pinus taeda, Sustentabilidade.

\section{ABSTRACT}

The aim was to evaluate the soil structural quality after forest harvesting by rubber-tires harvester and excavator hydraulic adapted in dystrophic Red Latosol (Oxisol). The experiment was designed in an entirely randomized manner with installation of four sampling units with three repetition points. In addition, soil samples were collected on the traffic path of the machine's strip road in the layers 0 to $0.10 ; 0.11$ to $0.20 ; 0.21$ to 0.30 ; and 0.31 to $0.40 \mathrm{~m}$ depth to determine the parameters of density and total porosity and also soil penetration resistance was measured on the strip road and inside strip road. Thus, it was possible to determine that the average density and total porosity before machine traffic were $1.10 \mathrm{~g} \mathrm{~cm}^{-3}$ and $53.85 \%$, respectively, with significant effects on these variables after the traffic of the rubber-tires harvester in all layers evaluated. As for soil penetration resistance, it was found that there was a significant increase in the strip road of the harvester, both rubber-tires and excavator, however, there was an increase of about $77 \%$ in the $0.10 \mathrm{~m}$ layer after traffic of the rubber-tires harvester.

KEYWORDS: Wood harvesting, Soil Physics, Pinus taeda, Sustainability. 


\section{INTRODUÇÃO}

A mecanização da colheita de madeira se intensificou significativamente no Brasil a partir da década de 1990, com a importação de máquinas de países com maior experiência florestal (MACHADO et al., 2014). Esse fato trouxe vários benefícios, como o aumento de produtividade, redução de custos de produção e maior segurança para os operadores de máquinas. No entanto, o aumento do tráfego de máquinas de grande porte em áreas florestais pode causar mudanças na qualidade estrutural do solo, assim como afetar as operações silviculturais subsequentes (REICHERT et al., 2018).

Diversos estudos foram realizados com o intuito de avaliar a relação entre máquinas florestais $e$ as propriedades físicas do solo (SAKAl et al., 2008; CAMBI et al., 2015). Entre os fatores que influenciam essa relação, destacam-se: matéria orgânica e textura do solo (BONETTI et al. 2017); teor de água (SOLGI \& NAJAFI, 2014); presença de biomassa residual da colheita de madeira (REICHERT et al., 2018); intensidade de tráfego (SILVA et al., 2008); carga por eixo (ANSORGE \& GODWIN, 2008); e tipos de rodados (GREČENKO \& PRIKNER, 2014).

Estudos sobre os efeitos do tipo de rodados das máquinas nos parâmetros físicos do solo apontam que esteiras ou pneus recobertos com semiesteiras podem mitigar os distúrbios causados ao solo (SAKAl et al., 2008). Tal fato deve-se a menor área de contato dos pneus em relação as esteiras, o que proporciona maior pressão e, consequentemente, maiores alterações na qualidade estrutural do solo (LAMANDÉ et al., 2018).

Em operações mecanizadas de colheita de madeira em sistema de toras curtas, geralmente utiliza-se o harvester no corte florestal, que pode ser de pneus ou esteiras (purpose built), bem como composto por uma escavadeira hidráulica adaptada com cabeçote harvester. Essa última opção é comumente utilizada em países da América do Sul e do Norte, Nova Zelândia, Reino Unido e Irlanda (LAITILA \& VÄÄTÄINEN, 2013), sendo vantajoso quanto ao custo de aquisição, disponibilidade à assistência técnica e peças de reposição, bem como menor custo operacional (SEIXAS \& BATISTA, 2014).

A escolha da máquina pelo tipo de rodado também pode ser influenciada pelos possíveis impactos que o tráfego proporciona ao solo (LAMANDÉ et al., 2018). Entretanto, são escassas as informações a respeito da qualidade estrutural do solo após o tráfego de harvester com diferentes tipos de rodados em condições similares de floresta, tipo de solo, matéria orgânica, textura e umidade, bem como quantidade de resíduos gerados na operação de colheita da madeira. Portanto, tais resultados podem auxiliar os gestores no planejamento das operações florestais, através da escolha de máquinas mais adequadas dentro do aspecto ambiental.

Neste contexto, objetivou-se quantificar os efeitos imediatos na qualidade estrutural do solo após o tráfego do harvester de pneus e escavadeira hidráulica adaptada em condições operacionais similares.

\section{MATERIAL E MÉTODOS}

O estudo foi realizado em uma floresta comercial localizada no município de Teixeira Soares, estado do Paraná, Brasil, nas coordenadas geográficas $25^{\circ} 19^{\prime} \mathrm{S}$ e $50^{\circ}$ $23^{\prime} \mathrm{W}$, com altitude média de $890 \mathrm{~m}$. O clima local é classificado, segundo Köppen, como temperado (Cfb), com precipitação média anual de $1.500 \mathrm{~mm}$, com ausência de estação seca e temperaturas médias de $18{ }^{\circ} \mathrm{C}$ e $22{ }^{\circ} \mathrm{C}$ nos meses mais frios e quentes, respectivamente (ALVARES et al., 2013).

Foi avaliado um povoamento de Pinus taeda L. nas operações de corte florestal submetido a corte raso aos 15 anos de idade, considerando o eito de corte composto por quatro linhas de plantio. As árvores apresentavam diâmetro à altura do peito médio de $0,27 \mathrm{~m}$, altura total média de 20,2 m e volume médio individual de 0,62 $\mathrm{m}^{3}$.

O solo foi classificado como LATOSSOLO VERMELHO distrófico típico (SANTOS et al., 2018). Para sua caracterização, a granulometria foi determinada pela metodologia descrita por Donagemma et al. (2017), enquanto a matéria orgânica foi caracterizada pelo método de Walkley-Black especificado por Pavan et al. (1992). A umidade gravimétrica no momento do tráfego foi determinada pela metodologia apresentada por Viana et al. (2017), que corresponde ao valor obtido durante a mensuração da resistência à penetração do solo.

O sistema de colheita de madeira utilizado foi o de toras curtas, onde as árvores foram derrubadas, processadas e empilhadas no interior do talhão e, posteriormente, extraídas para as margens das estradas. Foram avaliados um harvester de pneus da marca Komatsu, modelo 911.3 X3M com tração 6x6, peso de $25.000 \mathrm{~kg}$, potência de 185 kW e força de tração de 156 KN (Figura 1a); e uma escavadeira hidráulica da marca CAT, modelo 320D FM, adaptada com cabeçote processador, rodados de esteiras, peso de $26.900 \mathrm{~kg}$, potência de 117 kW e força de tração de 248 KN (Figura 1b). 

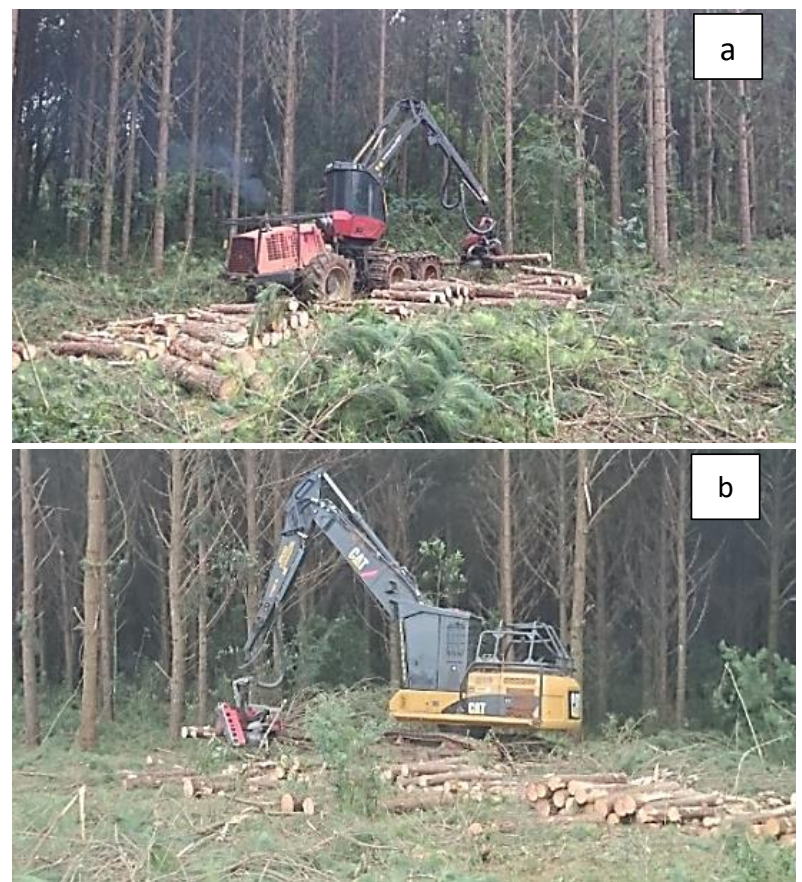

Figura 1. Máquinas avaliadas para as atividades de corte em povoamentos de Pinus taeda: (a) harvesters de pneus e (b) escavadeira hidráulica adaptada.

A seleção das máquinas avaliadas considerou a similaridade de peso, desconsiderando a potência do motor, pois, as operações do harvester são realizadas de forma estacionária a maior parte do tempo, sem a exigência de força de tração. Além disso, realizou-se o processamento das árvores na parte dianteira da máquina, dispondo os resíduos compostos pela porção final do fuste, galhos e acículas na linha de tráfego na mesma quantidade para ambas as máquinas, devido a homogeneidade do povoamento avaliado e toras produzidas.

Para avaliar a qualidade estrutural do solo, as máquinas trafegaram uma única vez sobre o solo durante a operação de corte florestal, efetuando-se a coleta de dados antes e após o tráfego do harvester de pneus e da escavadeira hidráulica. Para isso, foram instaladas quatro unidades amostrais (parcelas) com três pontos de repetição localizados na trilha de tráfego da máquina (rodados) e espaçados a $5 \mathrm{~m}$ entre si. Além disso, as amostras foram obtidas em quatro camadas de profundidades do solo: 0 a 0,10; 0,11 a 0,20; 0,21 a 0,30; e 0,31 a 0,40 m (Figura 2).

Para coleta de amostras indeformadas do solo, utilizouse um amostrador com anéis volumétricos de $86,35 \mathrm{~cm}^{3}$. Em seguida, as amostras foram enviadas para laboratório para determinação da densidade e da porosidade total do solo (ALMEIDA et al., 2017a; 2017b).

A resistência do solo à penetração foi avaliada com uso de um penetrômetro eletrônico digital, equipado com uma ponta cônica, com ângulo de vértice de $60^{\circ}$ e base de $1 \mathrm{~cm}^{2}$
(11,28 $\mathrm{mm}$ de diâmetro nominal), com velocidade de penetração máxima de $2 \mathrm{~cm} \mathrm{~s}^{-1}$. As leituras foram realizadas em seis pontos amostrais por parcela, sendo três pontos na linha de rodados ao longo da trilha de tráfego (rodados) e três entre elas (entre rodados) até a profundidade de 0,60 $\mathrm{m}$, com intervalos de 0,01 $\mathrm{m}$.

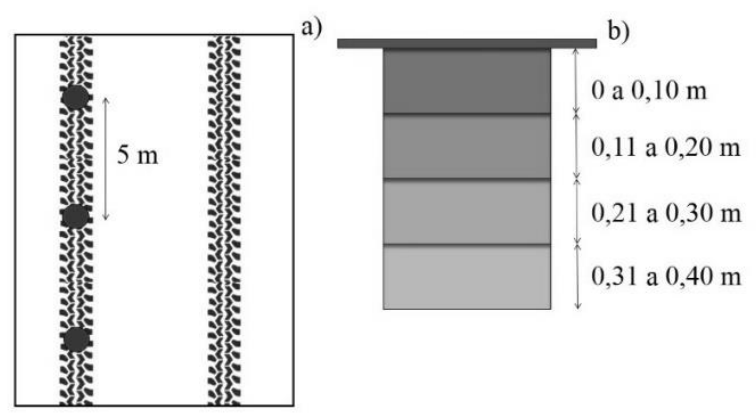

Figura 2. Esquema de coleta das amostras indeformadas: (a) Parcela com três pontos amostrais e b) profundidades obtidas para amostras do solo em cada ponto amostral.

Por meio de um delineamento inteiramente casualizado, considerou-se como tratamento os valores obtidos antes e após o tráfego das máquinas, enquanto para os valores de resistência do solo à penetração, também foram considerados os valores obtidos na linha de tráfego (rodados) e entre rodados. Os dados foram submetidos ao teste de normalidade de KolmogorovSmirnov $(\alpha \leq 0,05)$, seguido pelo teste de Bartlett para a verificação da homogeneidade das variâncias $(\alpha \leq 0,05)$ e análise de variância, com as médias comparadas pelo teste de Tukey $(\alpha \leq 0,05)$ para cada camada de profundidade avaliada.

\section{RESULTADOS E DISCUSSÃO}

O solo avaliado apresentou textura média muito argilosa, com umidade gravimétrica entre 0,43 e 0,37 kg kg 1 nas diferentes profundidades avaliadas (Tabela 1 ). Quanto a quantidade de matéria orgânica, observou-se uma variação decrescente em profundidade, com $46,9 \mathrm{~g} \mathrm{~kg}^{-1}$ na camada superficial (até $0,10 \mathrm{~m}$ ) e $33,5 \mathrm{~g} \mathrm{~kg}^{-1}$ na mais profunda $(0,31$ a $0,40 \mathrm{~m})$.

Os valores médios de densidade e porosidade total antes do tráfego das máquinas foram de $1,1 \mathrm{~g} \mathrm{~cm}^{-3} \mathrm{e}$ $53,85 \%$, respectivamente (Tabela 2 ). Após o tráfego do harvester de pneus, os efeitos foram significativos na densidade (média de $1,20 \mathrm{~g} \mathrm{~cm}^{-3}$ ) e porosidade total do solo (média de $49,77 \%$ ) em todas as camadas avaliadas. 
Por outro lado, esses parâmetros físicos do solo avaliados após o tráfego da escavadeira hidráulica não apresentaram diferença estatística significativa em relação aos valores obtidos antes do tráfego das máquinas (Tabela 2).

Tabela 1. Textura, umidade gravimétrica e matéria orgânica do solo.

\begin{tabular}{|c|c|c|c|c|c|}
\hline \multirow{2}{*}{ Prof. (m) } & \multicolumn{3}{|c|}{ Textura (\%) } & \multirow{2}{*}{$\begin{array}{c}\text { Ug } \\
\left(\mathrm{kg} \mathrm{kg}^{-1}\right)\end{array}$} & \multirow{2}{*}{$\begin{array}{c}\text { MO } \\
\left(\mathrm{g} \mathrm{kg}^{-1}\right)\end{array}$} \\
\hline & Areia & Silte & Argila & & \\
\hline $0,00-0,10$ & 14,8 & 28,6 & 56,6 & 0,43 & 46,9 \\
\hline $0,11-0,20$ & 15,5 & 24,5 & 60,0 & 0,41 & 40,2 \\
\hline $0,21-0,30$ & 12,0 & 26,0 & 62,0 & 0,39 & 40,2 \\
\hline $0,31-0,40$ & 14,9 & 21,2 & 63,9 & 0,37 & 33,5 \\
\hline Média & 14,3 & 25,1 & 60,6 & 0,40 & 40,2 \\
\hline
\end{tabular}

Ug é Umidade gravimétrica e MO é matéria orgânica.

O harvester de pneus ocasionou maiores valores de densidade do solo, possivelmente, em decorrência da menor distribuição da pressão dos rodados desta máquina sobre o solo em comparação com a escavadeira com rodados de esteiras. Isso se deve ao fato de que as alterações da qualidade estrutural do solo dependem da área de contato entre rodados e o solo, da pressão de insuflagem e da distribuição da força na área de contato (SAKAl et al., 2008; LAMANDÉ et al., 2018).

Os incrementos de densidade ocasionados pelo tráfego do harvester de pneus em relação aos valores obtidos antes do tráfego das máquinas foram maiores nas camadas mais profundas do solo (Tabela 2). Tais camadas do solo são mais suscetíveis a compactação, devido a menor proporção de matéria orgânica e de agregados do solo, o que proporciona uma tendência natural de elevação da densidade do solo. Consequentemente, a porosidade total apresentou tendência similar ao da densidade do solo, uma vez que possuem relações inversamente proporcionais entre elas, o que, também, é relatado por Lima et al. (2007).

A resistência do solo à penetração foi o parâmetro físico mais sensível quanto à qualidade estrutural do solo, principalmente, após o tráfego da escavadeira hidráulica adaptada, mostrando diferença estatística significativa com os valores obtidos antes do tráfego (Tabela 3). Entretanto, o harvester de pneus apresentou os maiores valores de resistência do solo à penetração. Os maiores valores foram obtidos nos pontos amostrados nas linhas de rodados quando comparados aos obtidos nos pontos entre rodados.

Tabela 2. Densidade e porosidade total em diferentes camadas do solo.

\begin{tabular}{cccc}
\hline $\begin{array}{c}\text { Prof. } \\
(\mathbf{m})\end{array}$ & $\begin{array}{c}\text { Antes do } \\
\text { tráfego das } \\
\text { máquinas }\end{array}$ & $\begin{array}{c}\text { Após } \\
\text { harvester de } \\
\text { pneus }\end{array}$ & $\begin{array}{c}\text { Após } \\
\text { escavadeira }\end{array}$ \\
\hline \multicolumn{4}{c}{ Densidade do solo $\left(\mathbf{g ~ c m}^{-3}\right)$} \\
\hline $0,00-0,10$ & $1,11 \mathrm{a}$ & $1,17 \mathrm{~b}$ & $0,99 \mathrm{a}$ \\
$0,11-0,20$ & $1,10 \mathrm{a}$ & $1,15 \mathrm{~b}$ & $1,05 \mathrm{a}$ \\
\hline $0,21-0,30$ & $1,05 \mathrm{a}$ & $1,21 \mathrm{~b}$ & $1,07 \mathrm{a}$ \\
\hline $0,31-0,40$ & $1,13 \mathrm{a}$ & $1,27 \mathrm{~b}$ & $1,16 \mathrm{a}$ \\
\hline Média & $\mathbf{1 , 1 0} \mathrm{a}$ & $\mathbf{1 , 2 0} \mathrm{b}$ \\
\hline \multicolumn{4}{c}{$\mathbf{1 , 0 7} \mathrm{a}$} \\
\hline $0,00-0,10$ & Porosidade total (\%) \\
\hline $0,11-0,20$ & $53,79 \mathrm{a}$ & $49,99 \mathrm{~b}$ & $58,54 \mathrm{a}$ \\
\hline $0,21-0,30$ & $51,52 \mathrm{a}$ & $51,52 \mathrm{~b}$ & $55,97 \mathrm{a}$ \\
\hline $0,31-0,40$ & $53,73 \mathrm{a}$ & $49,92 \mathrm{~b}$ & $55,79 \mathrm{a}$ \\
\hline Média & $53,85 \mathrm{a}$ & $47,65 \mathrm{~b}$ & $53,26 \mathrm{a}$ \\
\hline As & $\mathbf{4 9 , 7 7} \mathrm{b}$ & $\mathbf{5 5 , 8 9} \mathrm{a}$ \\
\hline
\end{tabular}

As médias nas linhas seguidas por letras distintas indicam diferenças significativas pelo teste de Tukey $(\alpha \leq 0,05)$.

Após o tráfego da escavadeira hidráulica adaptada na camada de 0 a 0,10 $\mathrm{m}$, a resistência do solo à penetração apresentou aumento médio de 39\%, enquanto após o tráfego do harvester de pneus, o aumento foi de $77 \%$ na mesma camada. Essa susceptibilidade na camada de 0 a $0,10 \mathrm{~m}$ de profundidade também foi relatada Silva et al. (2016). Além disso, estudo de Ampoorter et al. (2010) relatam que as alterações físicas do solo tendem a ser mais intensas logo abaixo da linha de tráfego das máquinas.

Tabela 3. Resistência à penetração do solo (Mpa) em diferentes profundidades do solo.

\begin{tabular}{|c|c|c|c|c|c|}
\hline \multirow{2}{*}{ Prof. (m) } & \multirow{2}{*}{$\begin{array}{l}\text { Antes do tráfego } \\
\text { das máquinas }\end{array}$} & \multicolumn{2}{|c|}{ Após harvester de pneus } & \multicolumn{2}{|c|}{ Após escavadeira } \\
\hline & & Rodados & Entre Rodados & Rodados & Entre Rodados \\
\hline $0,00-0,10$ & $0,61 \mathrm{a}$ & $0,85 \mathrm{~b}$ & $0,55 \mathrm{a}$ & $1,08 \mathrm{c}$ & $0,65 a$ \\
\hline $0,11-0,20$ & $1,20 \mathrm{a}$ & $1,45 b$ & $1,01 \mathrm{a}$ & $1,51 \mathrm{c}$ & $1,25 \mathrm{a}$ \\
\hline $0,21-0,30$ & $1,43 a$ & $1,59 \mathrm{~b}$ & $1,34 \mathrm{a}$ & $1,74 \mathrm{c}$ & $1,57 \mathrm{a}$ \\
\hline $0,31-0,40$ & $1,51 \mathrm{a}$ & $1,74 \mathrm{~b}$ & $1,60 \mathrm{a}$ & $1,87 \mathrm{c}$ & $1,91 \mathrm{a}$ \\
\hline $0,41-0,50$ & $1,82 \mathrm{a}$ & $1,75 \mathrm{~b}$ & $1,71 \mathrm{a}$ & $1,93 \mathrm{c}$ & $1,92 \mathrm{a}$ \\
\hline $0,51-0,60$ & 1,87 a & $2,00 \mathrm{~b}$ & $1,77 \mathrm{a}$ & $2,24 \mathrm{c}$ & $1,77 \mathrm{a}$ \\
\hline Média & $1,41 \mathrm{a}$ & $1,56 \mathrm{~b}$ & $1,33 \mathrm{a}$ & $1,73 \mathrm{c}$ & $1,51 \mathrm{a}$ \\
\hline
\end{tabular}

As médias nas linhas seguidas por letras distintas indicam diferenças significativas pelo teste de Tukey $(\alpha \leq 0,05)$. 
Considerando-se valores críticos da literatura, não se observou valores de resistência à penetração acima de 2 Mpa (Figura 3), sendo este o limite considerado potencialmente crítico em que pode começar a haver interferência ao desenvolvimento normal radicular das plantas (GREACEN \& SANDS, 1980; ZOU et al., 2000).
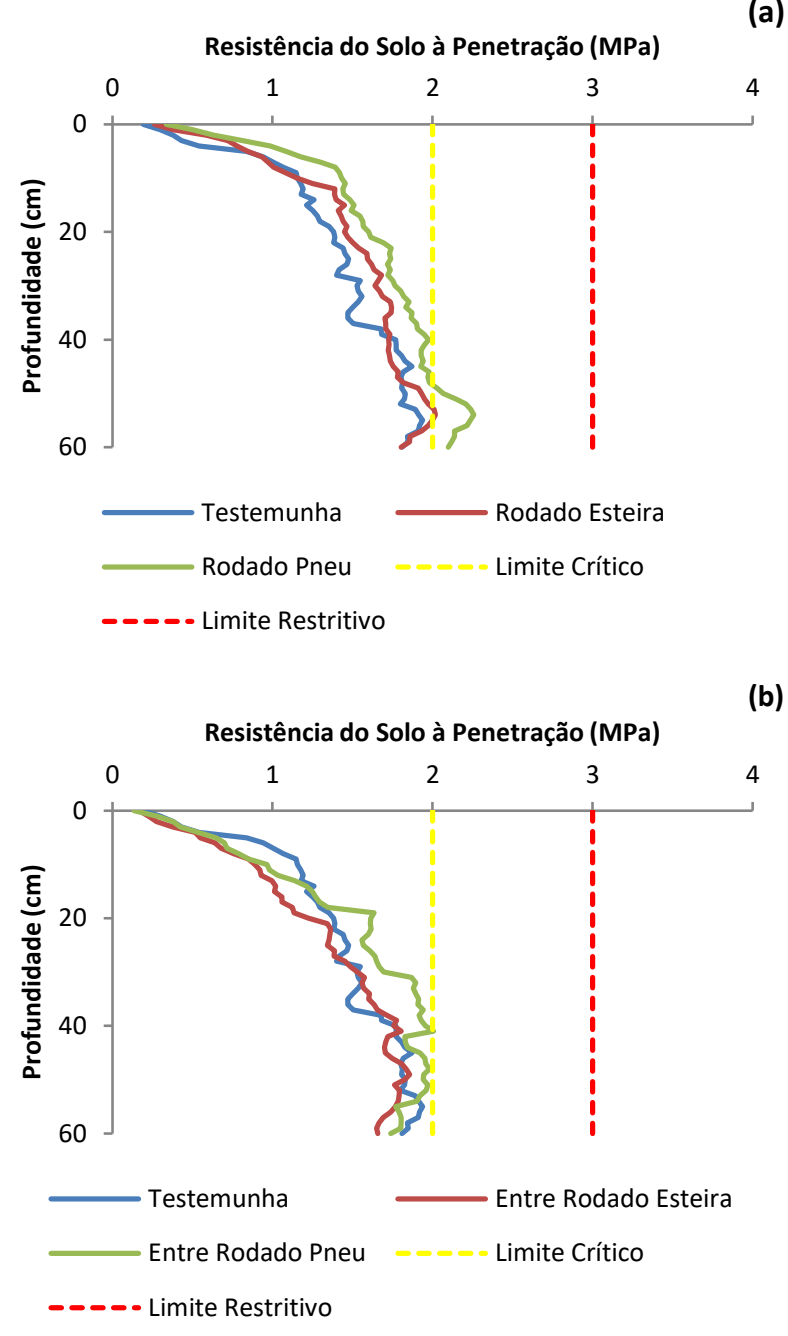

Figura 3. Valores médios de resistência do solo à penetração (MPa). a) Trilha dos rodados; e b) Trilha entre rodados. Linha tracejada de cor amarela indica valor de resistência à penetração crítica (Limite crítico) (GREACEN \& SANDS, 1980); e linha tracejada vermelha indica valor de resistência à penetração restritiva (Limite restritivo) (ZOU et al., 2000).

\section{CONCLUSÕES}

As alterações nos parâmetros físicos do solo foram significativas após o tráfego do harvester de pneus, sendo o solo menos suscetível ao tráfego da escavadeira hidráulica adaptada, sem diferença significativa na maioria dos parâmetros físicos avaliados em relação aos valores obtidos antes do tráfego das máquinas, sendo assim, recomenda-se seu uso nas condições do solo avaliado.

\section{REFERÊNCIAS}

ALMEIDA, B.G. et al. Densidade do solo, In: Manual de Métodos de Análise de Solo. Brasília: Embrapa, 2017a.

ALMEIDA, B.G. et al. Porosidade. In: Manual de Métodos de Análise de Solo. Brasília: Embrapa, 2017b.

ALVARES, C.A. et al. Köppen's climate classification map for Brazil. Meteorologische Zeitschrift, v.22, n.6, p.711-728, 2013.

AMPOORTER, E. et al. Assessing the effects of initial soil characteristics, machine mass and traffic intensity on forest soil compaction. Forest Ecology and Management, v.260, n.10, p.1664-1676, 2010.

ANSORGE, D.; GODWIN, R.J. The effect of tyres and a rubber track at high axle loads on soil compaction - Part 2: Multi-axle machine studies. Biosystems Engineering, v.99, n.3, p.338-347, 2008.

BONETTI, J.A. et al. Resilience of soils with different texture, mineralogy and organic matter under long-term conservation systems. Soil and Tillage Research, v.174, p.104-112, 2017.

CAMBI, M. et al. The impact of heavy traffic on forest soils: A review. Forest Ecology and Management, v.338, p.124-138, 2015.

DONAGEMMA, G.K. et al. Análise granulométrica. In: Manual de Métodos de Análise de Solo. Brasília: Embrapa, 2017.

GREACEN, E.L.; SANDS, R. Compaction of forest soils: a review. Australian Journal of Soil Research, v.18, p.163-189, 1980.

GREČENKO, A.; PRIKNER. P. Tire rating based on soil compaction capacity. Journal of Terramechanics, v.52, p.77-92, 2014.

LAITILA, J.; VÄÄTÄINEN, K. The cutting productivity of the Excavator-based harvester in integrated havesting of Pulpwood and Energy Wood. Baltic Forest, v.19, n.2, p.289-300, 2013.

LAMANDÉ, M. et al. Risk assessment of soil compaction in Europe - Rubber tracks or wheels on machinery. Catena, v.167, p.353362, 2018.

LIMA, C.G.R. et al. Correlação linear e espacial entre a produtividade de forragem, a porosidade total e a densidade do solo de Pereira Barreto (SP). Revista Brasileira de Ciência do Solo, v.31, n.6, p.1233-1244, 2007.

MACHADO, C.C. et al. O setor florestal brasileiro e a colheita florestal, In: Colheita Florestal, Viçosa: UFV, 2014.

PAVAN, M. A. et al. Manual de análise química de solo e controle de qualidade, Londrina, PR: IAPAR, 1992.

REICHERT, J.M. et al. Ground-based harvesting operations of Pinus taeda affects structure and pore functioning of clay and sandy clay soils. Geoderma, v.331, p.38-49, 2018.

SAKAI, H. et al. Soil compaction on forest soils from different kinds of tires and tracks and possibility of accurate estimate. Croatian Journal of Forest Engineering, v.29, n.1, p.15-27, 2008. 
SANTOS, H.G. et al. Sistema Brasileiro de Classificação de Solos, Brasília: Embrapa, 2018.

SEIXAS, F.; BATISTA, J.L.F. Technical and economical comparison between wheel harvesters and excavators. Ciência Florestal, v.24, n 1, p.185-191, 2014.

SILVA, R.B. et al. Contact pressures and the impact of farm equipment on Latosol with the presence and absence of sugarcane straw. Ciência e Agrotecnologia, v.40, n.3, p.265-278, 2016.

SILVA, S.R. et al. Soil compaction and eucalyptus growth in response to forwarder traffic intensity and load. Revista Brasileira de Ciências do Solo, v.32, n.3, p.921-932, 2008.

SOLGI, A.; NAJAFI, A. The impacts of ground-based logging equipment on forest soil. Journal of Forest Science, v.60, n.1, p.28-34, 2014.

VIANA, J.H.M. et al. Umidade atual. In: Manual de Métodos de Análise de Solo, Brasília: Embrapa, 2017.

ZOU, C. et al. Least limiting water range: A potential indicator of physical quality of forest soils. Australian Journal of Soil Research, v.28, p.947-958, 2000.

Recebido em 04-08-2021 Aceito em 09-09-2021 